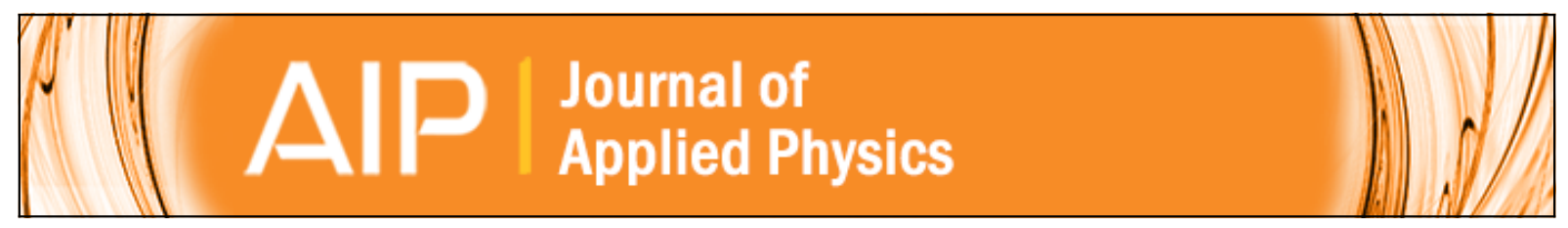

Investigation of external noise and its rejection in magnetoelectric sensor design

Zengping Xing, Junyi Zhai, Jiefang Li, and Dwight Viehland

Citation: Journal of Applied Physics 106, 024512 (2009); doi: 10.1063/1.3176500

View online: http://dx.doi.org/10.1063/1.3176500

View Table of Contents: http://scitation.aip.org/content/aip/journal/jap/106/2?ver=pdfcov

Published by the AIP Publishing

Articles you may be interested in

A pencil-like magnetoelectric sensor exhibiting ultrahigh coupling properties

J. Appl. Phys. 113, 134101 (2013); 10.1063/1.4798509

Shifting the operating frequency of magnetoelectric sensors

J. Appl. Phys. 111, 07 C714 (2012); 10.1063/1.3677840

Comparison of noise floor and sensitivity for different magnetoelectric laminates

J. Appl. Phys. 108, 084509 (2010); 10.1063/1.3486483

Noise and scale effects on the signal-to-noise ratio in magnetoelectric laminate sensor/detection units Appl. Phys. Lett. 91, 182902 (2007); 10.1063/1.2804118

Extremely low frequency response of magnetoelectric multilayer composites

Appl. Phys. Lett. 86, 102901 (2005); 10.1063/1.1881784

MIT LINCOLN

LABORATORY

CAREERS

Discover the satisfaction of innovation and service

to the nation
- Space Control

- Air \& Missile Defense

- Communications Systems \& Cyber Security

- Intelligence, Surveillance and

Reconnaissance Systems

\section{LINCOLN LABORATORY}

MASSACHUSETTS INSTITUTE OF TECHNOLOGY

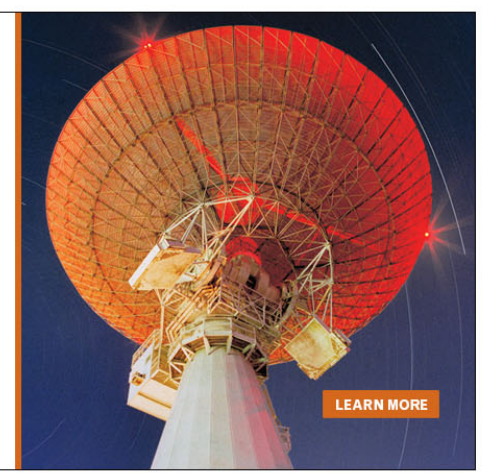




\title{
Investigation of external noise and its rejection in magnetoelectric sensor design
}

\author{
Zengping Xing, ${ }^{\text {a) }}$ Junyi Zhai, Jiefang Li, and Dwight Viehland \\ Department of Materials Science and Engineering, Virginia Tech, Blacksburg, Virginia 24061, USA
}

(Received 16 March 2009; accepted 16 June 2009; published online 28 July 2009)

\begin{abstract}
Laminated magnetoelectric (ME) composites consisting of magnetostrictive and piezoelectric layers are an important class of magnetic sensors. Here, we will present a means to design ME sensors with in-built capabilities to cancel environmental noise, based on designing the sensor signal and the noise in different fundamental modes. We then show that some composite designs offer unique capabilities to reject acoustic or thermal noise sources. (C) 2009 American Institute of Physics. [DOI: 10.1063/1.3176500]
\end{abstract}

\section{BACKGROUND}

The magnetoelectric (ME) effect is defined by the electric field $(E)$ induced under application of a magnetic field $(H)$; or vice versa, by the magnetic induction $(B)$ induced under application of an electric field $(E) .{ }^{1}$ Generally, the ME response of a material is described by the ME voltage coefficient $\left(\alpha_{\mathrm{ME}}\right)$, defined as ${ }^{2}$

$$
\alpha_{\mathrm{ME}}=E / H \text {. }
$$

Since 2000, dramatically enhanced values of $\alpha_{\mathrm{ME}}$ have been found in laminated composites consisting of magnetostrictive and piezoelectric layers epoxied together. ${ }^{3-10}$ The said laminated ME composites have ME voltage coefficients much larger than any other ME materials. It has accordingly been designated as a giant $\mathrm{ME}$ effect. The mechanism behind the giant ME effect is easy to understand. When $H$ is applied to the magnetostrictive layer, a strain is generated in that layer; then, this strain is transferred to the piezoelectric layer by elastic bonding to the magnetostrictive one; and finally an electric field is generated across the piezoelectric layer via piezoelectricity.

There have been many investigations concerning $\mathrm{ME}$ composite configurations and the poling (magnetization) direction with respect to the long axis of the composite layers. There are many different ways to put ME laminate composites together of various texture symmetries. We can identify four basic modes of operation in a long axis laminate, and subsequently other more complex designs. The basic modes are (a) longitudinally magnetized and longitudinally poled or (L-L) mode, (b) longitudinally magnetized and transversely poled or (L-T) mode, (c) transversely magnetized and longitudinally poled or (T-L) mode, and (d) transversely magnetized and transversely poled or (T-T) mode. ${ }^{7}$ Some of the more complex designs are (e) unimorph, ${ }^{11}$ (f) bimorph structures, ${ }^{8}$ and (f) symmetrically poled or push-pull configurations. ${ }^{12}$ These various modes are illustrated in Figs. 1(a)-1(f), respectively.

Because of their large values of $\alpha_{\mathrm{ME}}$, laminate ME composites with giant magnetoelectricity are potentially useful

\footnotetext{
a)Tel.: (+1) (540) 231 6928. FAX: (+1) (540) 231 8919. Electronic mail: xing@vt.edu.
}

for the applications in magnetic and electric current sensors. As in the case of all sensors, noise is a very important factor that limits the sensitivity. We can classify the noise sources into internal and external ones. The internal noise, sometimes was referred to intrinsic noise, comes from random phenomenon of nature: such as thermal agitation of electrons in a resistor, generation and recombination of electron-pole pairs in a semiconductor, etc. ${ }^{13}$ For ME sensor design optimization, in considering the internal noise, it is very important to build physical and electrical models with noise included. ${ }^{14}$ Previously, we have investigated two methods, scale effect and ME array, by which to increase the sensitivity of ME laminate sensors. ${ }^{15,16}$ However, practical sensor applications are always notably affected by the environmental or external noise sources, such as thermal fluctuations and mechanical vibrations. In ME sensors, thermal fluctuation noise is pyroelectric in origin, where the spontaneous polarization of the piezoelectric phase is temperature dependent, resulting in a dielectric displacement current in response to temperature changes; whereas the vibrational noise is piezoelectric in origin, where the spontaneous polarization is coupled to pressure and stress changes, via piezoelectricity. As for all magnetic field sensors, it is important that ME sensors be designed by such a means that optimizes its abilities to cancel these external noise.

Here, we consider the ability of various ME modes to cancel environmental noise from thermal and vibrational sources. The findings are summarized in Table I, where it can be seen that there are various configurations that have the

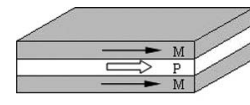

(a) L-L

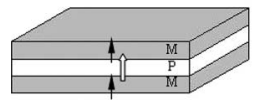

(d) T-T

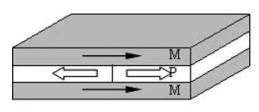

(g) L-L push-pull

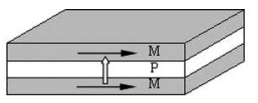

(b) L-T

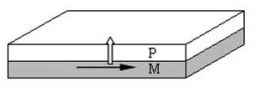

(e) Unimorph

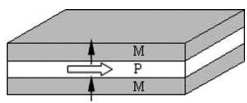

(c) T-L

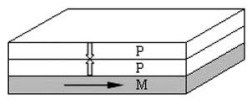

(f) Bimorph
FIG. 1. Structures of (a) L-L, (b) L-T, (c) T-L, (d) T-T, (e) unimorph, (f) bimorph, and (g) push-pull. 
TABLE I. Noise cancellation summary.

\begin{tabular}{lcc}
\hline \hline Mode & Vibration cancellation & Thermal cancellation \\
\hline Unimorph & No & No \\
Bimorph & Partially & Partially \\
L-T, T-T & Yes & No \\
L-L, T-L & Yes & No \\
Push-pull & Yes & No \\
Multipush-pull & Yes & No \\
SS-UN & Yes & No \\
U.S.-SN $^{\text {b }}$ & No & Yes \\
\hline
\end{tabular}

${ }^{\mathrm{a}}$ SS-UN: Symmetrical-signal, unsymmetrical noise.

${ }^{b}$ U.S.-SN: Unsymmetrical-signal, symmetrical noise.

abilities to reject thermal or acoustic noise. The results provide insights into how future ME laminates configurations might be arranged in order to provide enhanced signal-tonoise ratios (SNRs).

\section{EXTERNAL NOISE ON ME SENSORS}

\section{A. Vibration}

Let us assume a rectangular ME laminate sensor, as shown in Fig. 2(a). Stray vibrations will excite various vibrational modes, but the dominant one will be the fundamental bending mode. It has the lowest resonance frequency, and thus will couple best to the environmental vibrational noise which is also generally of low frequencies. For simplicity, we consider here that vibration noise excites only this bending mode.

In Fig. 2(b), we illustrate the fundamental bending mode for a rectangular ME sensor. Please note that there is a neutral surface, which ideally has no shear force exerted on it. The area of sensor that is below this neutral surface is in compression, whereas that above it is in tension, or vice versa. If we measure the piezoelectrically induced charge both above and below the neutral line, we would find that they are of opposite signs but nearly of the same magnitude. This figure conceptually demonstrates that vibrational acoustic noise sources can be cancelled, if the structure of the sensor is design to be mechanically symmetrical.

\section{B. Temperature fluctuation}

Temperature is a scalar property. The four basic ME modes and the push-pull mode illustrated in Fig. 1 have symmetric structures. When the temperature of a symmetrical rectangle is changed, thermal expansion will strain it by the

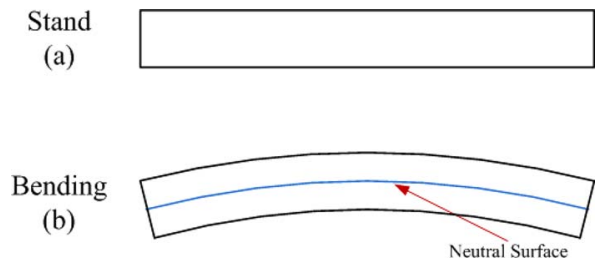

FIG. 2. (Color online) (a) A force free rectangular plate and (b) influence of vibrations on the shape deformation of the rectangular plate. (a)
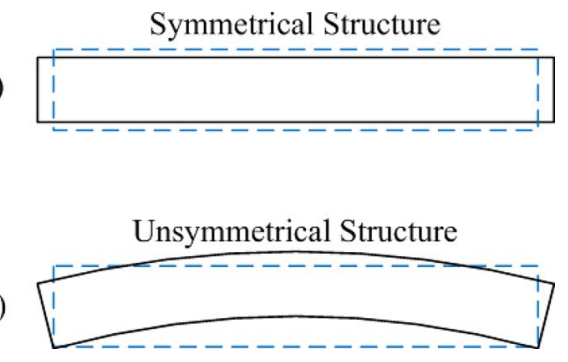

FIG. 3. (Color online) External thermal noise on the plate in a (a) symmetrical structure and (b) unsymmetrical structure (dashed line: standing; solid line: reaction to external noise).

same percent in the same directions, as shown in Fig. 3(a). In this case, only longitudinal/transverse deformations will be excited.

However, the more complex ME laminate configurations shown in Figs. 1(e) and 1(f) are nonsymmetric. Thus, when the temperature changes, both bending and longitudinal modes will be excited due to thermal expansion differences between the various layers, as illustrated in Fig. 3(b). Again, there is a neutral surface for the induced bending mode. Charges induced from this temperature change from above and below the neutral line are of opposite sign but nearly the same magnitude. This figure conceptually demonstrates that thermal noise can be partially cancelled by the sensor itself by use of an unsymmetrical structure.

\section{ANALYSIS OF EXTERNAL NOISE IN DIFFERENT MODES OF ME LAMINATES}

In this section, the noise cancellation ability refers to the capability that the design of the sensor can cancel the noise while keep the signal unchanged, or cancel the noise much more than the signal does. The SNR will increase accordingly.

\section{A. Unimorph}

A unimorph is an unsymmetrical structure. Its ME signal will contain both expanding and bending mode contributions. In this case, vibrational bending noise and the signal bending mode will be mixed-we will be unable to separate them. Accordingly, a unimorph ME laminate will not have vibration noise cancellation ability. In a similar manner, external thermal noise does not cancel for a unimorph structure, again simply because the signal and noise modes are mixed. Figure 4 illustrates the output charge induced across the piezoelectric layer due to stray vibrations and temperature fluctuation.

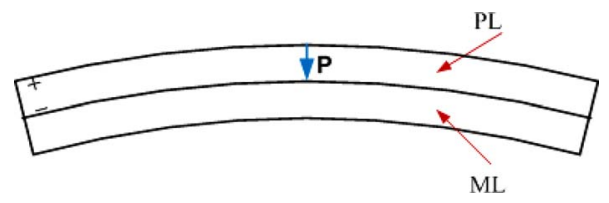

FIG. 4. (Color online) Effect of vibrational and thermal noise on a unimorph (PL: piezoelectric layer; ML: magnetostrictive layer). 
(a)

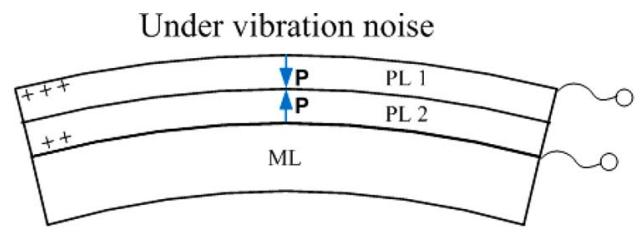

(b)

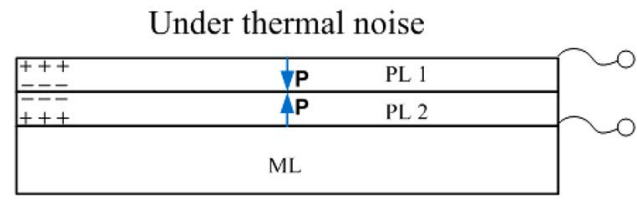

(c)

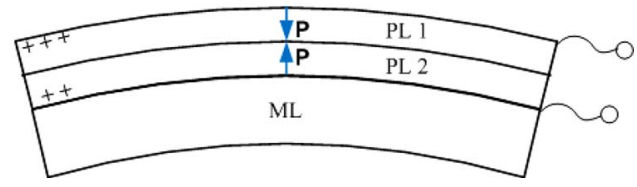

FIG. 5. (Color online) Effect of environmental noise on bimorph ME laminate (a) vibrational noise which excites bending mode; (b) thermal noise which excites a longitudinal mode; and (c) thermal noise which excites a bending mode.

\section{B. Bimorph}

A bimorph is also an unsymmetrical structure. The ME signal will again produce both bending and expanding mode contributions. In this case, the expanding mode signal output will cancel due to the structure's design, and consequently only the bending signal mode output will remain. This bending mode signal is analyzed in Fig. 5(a). It has a differential structure, which will result in a partial cancellation of the noise introduced by the vibration.

External thermal noise will excite both expanding and bending modes in a bimorph. Analysis of the noise introduced by thermal changes is given in Fig. 5(b) for the expanding mode and in Fig. 5(c) for the bending one. We can see in (b) that thermal noise is fully cancelled in the expanding mode, but in (c) that it is only partially cancelled in the bending mode.

The combinations of Figs. 5(a)-5(c) demonstrate that the bimorph ME structure has the ability to partially cancel both external vibrational and thermal noises. However, unfortunately, at the same time, this cancellation will also reduce the ME signal. The further the distance of the two piezoelectric transducer layers from the neutral surface, the smaller the remaining signal will be. Accordingly, practical designs must consider both the noise cancellation and signal loss.

\section{T mode (L-T, T-T)}

The $\mathrm{T}$ mode configuration has a symmetrical structure. For this configuration, a ME signal is produced only in the expanding mode, whereas vibrational noise is introduced through the bending mode. This configuration has a built-in ability to cancel vibrations in the bending mode. As shown in Fig. 6(a) (due to the $\mathrm{T}$ mode structure's symmetric nature, only the piezolayers portion of the ME laminate is shown), (a)

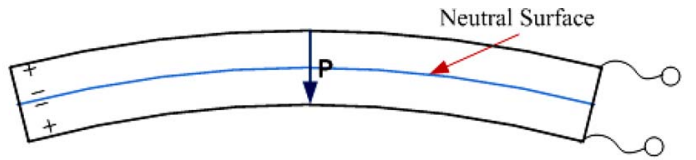

(b)

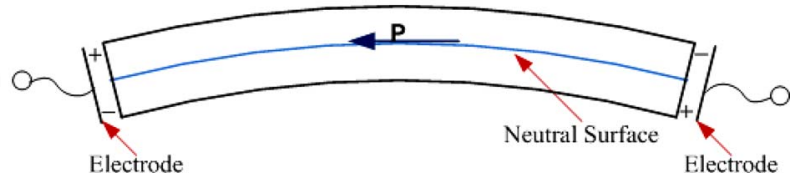

FIG. 6. (Color online) Effect of vibration bending noise on the PZT layer in (a) L-T, T-T modes ME laminate and (b) L-L, T-L modes ME laminate.

the sign of the induced charges are reversed on going from above to below the neutral surface. Accordingly, the net output from vibrational noise is rejected.

Due to the symmetric nature of the T mode configuration, a bending mode will not be excited by thermal changes. Rather, thermal noise will be constrained to the expanding mode. This makes it impossible to separate the thermal noise from the ME signal by the sensor itself.

\section{L mode (L-T, T-L)}

The $\mathrm{L}$ mode configuration is also a symmetrical structure. Again, the ME signal is produced only in the expanding mode. Only vibrational noise, and not thermal, can excite a bending mode which can be separated from the expanding mode excited by the ME signal. Analysis of the vibrational noise is illustrated in Fig. 6(b) (due to the L mode structure's symmetric nature, only the piezolayer portion of the ME laminate is shown). Again, there is a neutral surface, above which there is a positive charge and below which there is a negative one; bending noise is cancelled out. However, because thermal noise is constrained to the expanding mode with the ME signal, it cannot be rejected without canceling the signal.

\section{E. Push-pull: Single unit}

Both the L-L and L-T push-pull configurations are symmetrical structures, where thermal noise again cannot excite a bending mode. These configurations are shown in Figs. 7 (a) and 7(b), respectively. In both of these push-pull modes, vibration noise is contained in the bending mode, whereas thermal noise in the expanding. Both structures have built-in vibration cancellation; as illustrated in the insets of the figures, where we can see that the output will be "0" for bending noise.

\section{F. Push-pull: Multiunit}

A modification of the L-L push-pull mode has been studied in magnetostrictive/(1-3)piezofiber composites, where the piezofibers are poled in a manner such as to create numerous push-pull units back to back. ${ }^{17}$ The poling condition of the piezofiber layer is illustrated in Fig. 8. This multipushpull structure also has the ability to cancel vibration noise, as shown in the inset of the figure. The advantage of this configuration is the higher geometric capacitance of the piezofi- 
(a)

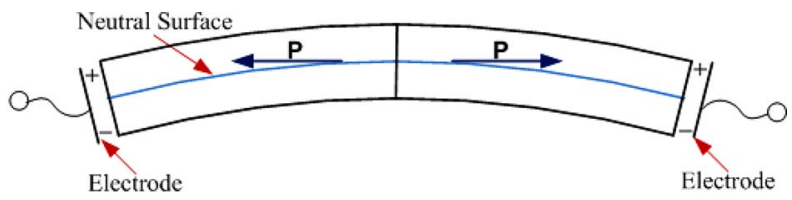

(b)

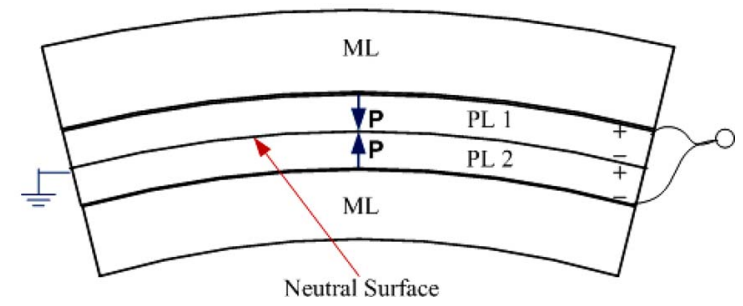

FIG. 7. (Color online) Vibration bending noise in (a) the L-L push-pull structure; and (b) the L-T push-pull structure, where the inset shows the cancellation of vibration effects.

ber layer, over that of a single monolithic one. Again, because of the symmetric nature of the structure, the thermal noise cannot be separated from the signal.

\section{G. Symmetrical Signal, unsymmetrical noise mode}

A symmetrical signal, unsymmetrical noise mode, (i.e., a SS-UN mode), is a ME construction that is designed to have mechanically symmetric responses to an applied signal, while having unsymmetrical responses to external noise. Following this designation, we can see that L-T, L-L, T-T, T-L, and push-pull modes all fall in this category. More accurately, we should note that these modes are only unsymmetrical to vibration noise, and not to thermal fluctuation. From our above analysis, it can be noted that thermal noise in a symmetrical structure can only excite a symmetrical deformation: i.e., the SS-UN mode cannot separate the thermal noise from the signal "itself." So for this mode design, when the temperature fluctuation is a problem, a high pass filter is required in the detection circuitry since temperature fluctuation is always a low frequency noise. Our previous research used this method to reduce the temperature's affect and stabilized the ME unit output. ${ }^{18}$

\section{H. Unsymmetrical signal, symmetrical noise mode}

An unsymmetrical signal, symmetrical noise mode, (i.e., a U.S.-SN mode), is a design where the sensor has mechanically unsymmetrical responses to an applied signal, while having symmetrical responses to external noise. An unsymmetrical signal configuration can be designed by introducing a nonmagnetic material, which has the same elastic stiffness

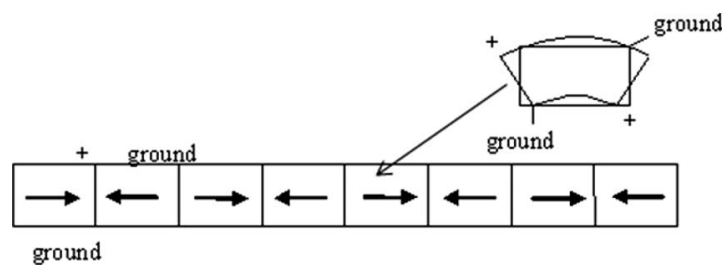

FIG. 8. Vibration bending noise in a multipush-pull configuration. Each individual push-pull unit works in the L-L mode. The inset shows the analysis of charges induced by bending. (a)

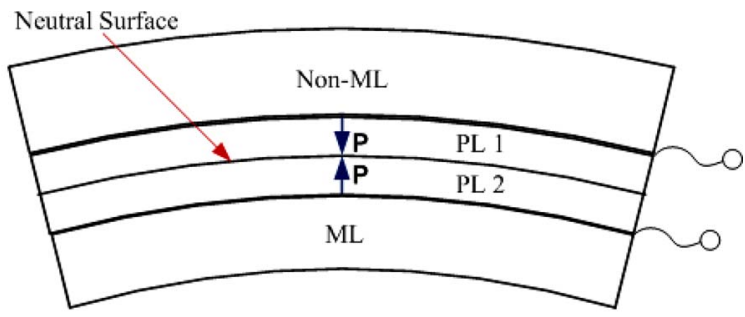

(b)

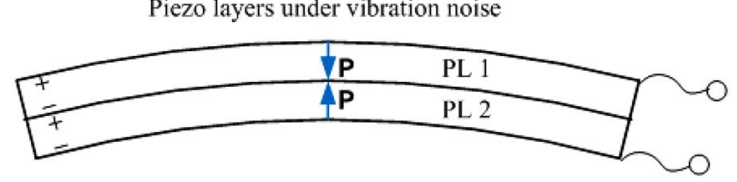

Piezo layers under thermal noise

\begin{tabular}{|l|ll|}
\hline+++ & $P$ & PL 1 \\
\hline--+ & $P$ & PL 2 \\
\hline+++ & $P$ & $P$
\end{tabular}

FIG. 9. (Color online) Bending on U.S.-SN structure 1 (a) and noise analysis of piezoelectric layer for the structure 1, (b) vibration bending noise, and (c) thermal noise.

and temperature coefficient as that of the magnetic layer(s). In this case, the magnitude of the signal will be decreased some, but will have advantages with regards to thermal noise cancellation. We illustrate this concept by the following designs.

U.S.-SN configuration 1 is illustrated in Fig. 9(a). It is a three layer structure consisting of a bimorph piezoelectric layer sandwiched between magnetostrictive and nonmagnetic layers. Analysis of the charges across the piezoelectric layer induced from bending is shown in Fig. 9(b). We can see that this configuration does not cancel vibration noise. Analysis of the charges induced across the piezoelectric layer by thermal changes is shown in Fig. 9(c). Assuming that the nonmagnetic and magnetostrictive layers have the same elastic stiffness and temperature coefficient thereof, we can see that the charge outputs cancel. Thus, the thermal noise is rejected.

U.S.-SN configuration 2 is illustrated in Fig. 10(a). It is a four layer structure consisting of two piezoelectric layers poled in the same direction sandwiched between magnetostrictive and nonmagnetic layers. However, the top electrode of PZT 1 and the bottom electrode of PZT 2 are connected as electrode 1, whereas the bottom electrode of PZT 1 and the top electrode of PZT 2 are connected as electrode 2. Analysis of the charges across the piezoelectric layer induced from this bending is shown in Fig. 10(b). We can see that this configuration does not cancel vibration noise. Analysis of the charges induced across the piezoelectric layer by thermal changes is shown in Fig. 10(c). Assuming that the nonmagnetic and magnetostrictive layers have the same elastic stiffness and temperature coefficient thereof, we can see that the charge outputs cancel. Thus, the thermal noise is rejected.

An unsymmetrical signal configuration could also be designed by making use of the piezomagnetic properties of the magnetostrictive layer. Previous research has shown that giant magnetostrictive materials (Terfenol-D, Metglass) require a dc bias to achieve a high effective piezomagnetic condi- 
(a)

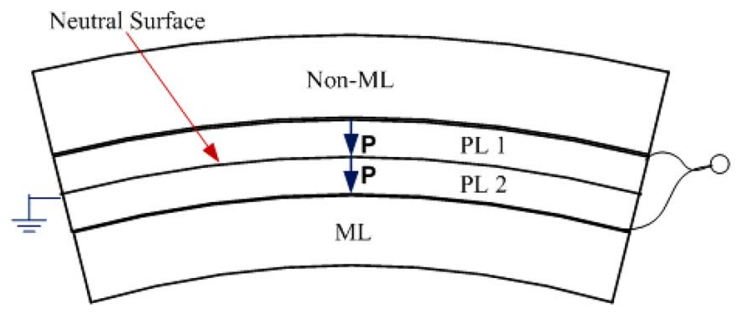

(b)

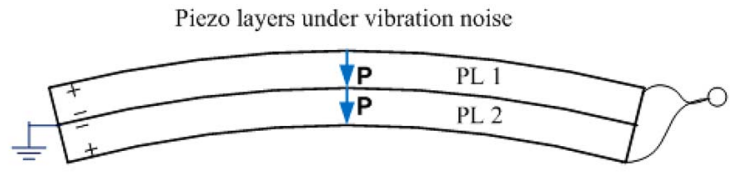

(c)

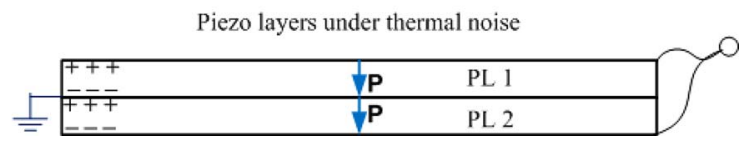

FIG. 10. (Color online) Bending on U.S.-SN structure 2 (a) and noise analysis of piezoelectric layer for the structure 2, (b) vibration bending noise, and (c) thermal noise.

tion, where the magnetization direction is determined by the sign of applied dc bias. If two magnetostrictive layers are magnetized in opposing directions, then accordingly their ME response will be opposite to each other. If we replace the nonmagnetostrictive layer in Fig. 9 (or Fig. 10) with a magnetostrictive one while making its magnetization direction opposite to the first, then the signal will be unsymmetrical, whereas the thermal noise response is symmetric. This structure is shown in Fig. 11 and has been shown to resist temperature fluctuation. Compared to Fig. 9, this structure will have a higher ME performance due to the top and the bottom magnetostrictive phase will both contribute for $\mathrm{ME}$ coupling. ${ }^{8}$

\section{INVESTIGATIONS OF VIBRATIONAL NOISE REJECTION}

Investigations of vibrational noise were performed for various ME sensors. Our experiment for vibration damping is illustrated in Fig. 12. The ME sensor was put on a small table above a shaker which was driven by a bipolar power amplifier (HP6826A); and a frequency dependent source signal, which was provided by a lock-in amplifier (SR850), was fed into the HP6826A for amplification. The vibration output signal was then measured by a SR850 Lock-in amplifier. The ME laminate composites that we characterized are listed in Table II, along with their corresponding size.

The vibration frequency dependence for these ME laminate sensors are shown in Fig. 13. We amplify the low frequency portion of the spectrum to better illustrate vibration

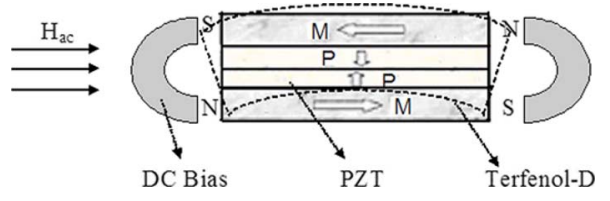

FIG. 11. (Color online) U.S.-SN mode 3.

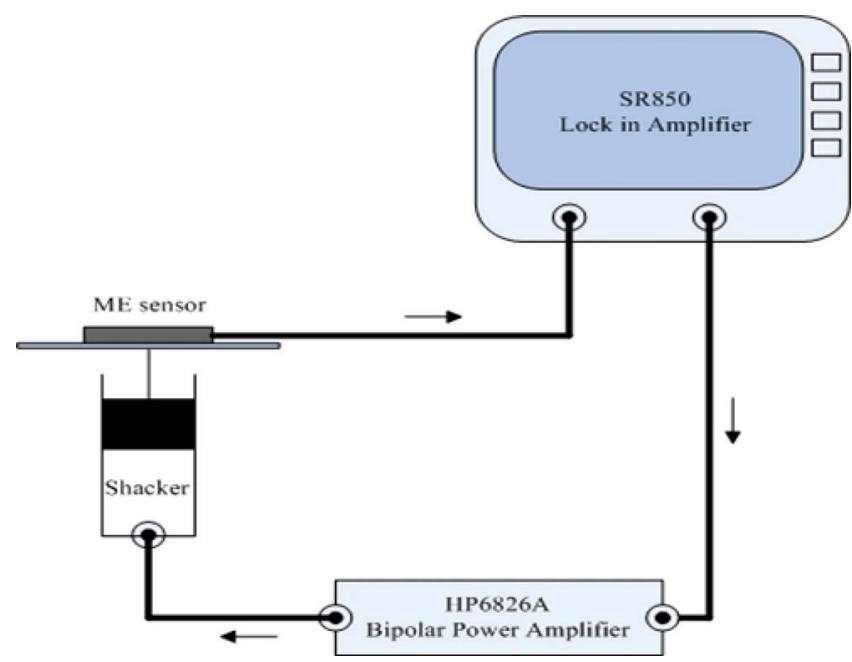

FIG. 12. (Color online) Vibrating damping experiment setup for ME laminate sensor.

noise rejection. In the figure inset, we can see that the L-T and LT-push-pull modes have the best vibration cancellation performance, while the bimorph has some capabilities in this regard, whereas the unimorph has nearly none. These findings are consistent with the predictions of Sec. III.

We next measured the ME voltage coefficient of these various ME laminate configurations of Table II at different dc bias. The results are shown in Fig. 14(a). We can see from this figure that the L-T mode has the highest ME induced voltage, compared to the bimorph and unimorph modes. For optimized dc biases for each of these ME sensors, we finally calculated the SNR, as shown in Fig. 14(b). We can see that the L-T and LT-PP modes have the highest value of SNR, while the bimorph is intermediate, whereas the unimorph has the lowest. Again, these results are consistent with our prediction in Sec. III.

\section{SUMMARY}

Our study has identified several principles for the design of magnetic sensors based on ME laminated composites that have enhanced capabilities to reject external noise. We introduced a concept of signal and noise modes and provided some rules for optimization. First, if the mode produced by the ME signal is the same as that made by noise, then the ME signal and noise cannot be separated. Only filters or magnetic gradiometers might help in this case, but then only

TABLE II. ME laminate sensor.

\begin{tabular}{lccc}
\hline \hline & $\begin{array}{c}\text { Each TD size } \\
L \times W \times H\left(\mathrm{~mm}^{3}\right)\end{array}$ & $\begin{array}{c}\text { Each PZT size } \\
L \times W \times H\left(\mathrm{~mm}^{3}\right)\end{array}$ & $\begin{array}{c}\text { Total size } \\
L \times W \times H\left(\mathrm{~mm}^{3}\right)\end{array}$ \\
\hline Mode and no. & $15 \times 6 \times 1.0$ & $15 \times 6 \times 2.2$ \\
Unimorph 1 & $14 \times 6 \times 1.2$ & $15 \times 6 \times 6.0$ & $15 \times 6 \times 3.4$ \\
Unimorph 2 & $14 \times 6 \times 2.4$ & $15 \times 6 \times 1.0$ & $15 \times 6 \times 2.2$ \\
Bimorph 1 & $14 \times 6 \times 1.2$ & $15 \times 6 \times 0.5$ & $15 \times 6 \times 3.4$ \\
Bimorph 2 & $14 \times 6 \times 2.4$ & $15 \times 6 \times 0.5$ & $15 \times 6 \times 2.2$ \\
LT 1 & $14 \times 6 \times 0.6$ & $15 \times 6 \times 1.0$ & $15 \times 6 \times 3.4$ \\
LT 2 & $14 \times 6 \times 1.2$ & $15 \times 6 \times 1.0$ & $15 \times 6 \times 2.2$ \\
LT-PP 1 & $14 \times 6 \times 0.6$ & $15 \times 6 \times 1.0$ & $15 \times 6 \times 3.4$ \\
LT-PP 2 & $14 \times 6 \times 1.2$ & $15 \times 6 \times 1.0$ &
\end{tabular}

${ }^{\mathrm{a}}$ LT-PP: LT-Push-pull mode. 


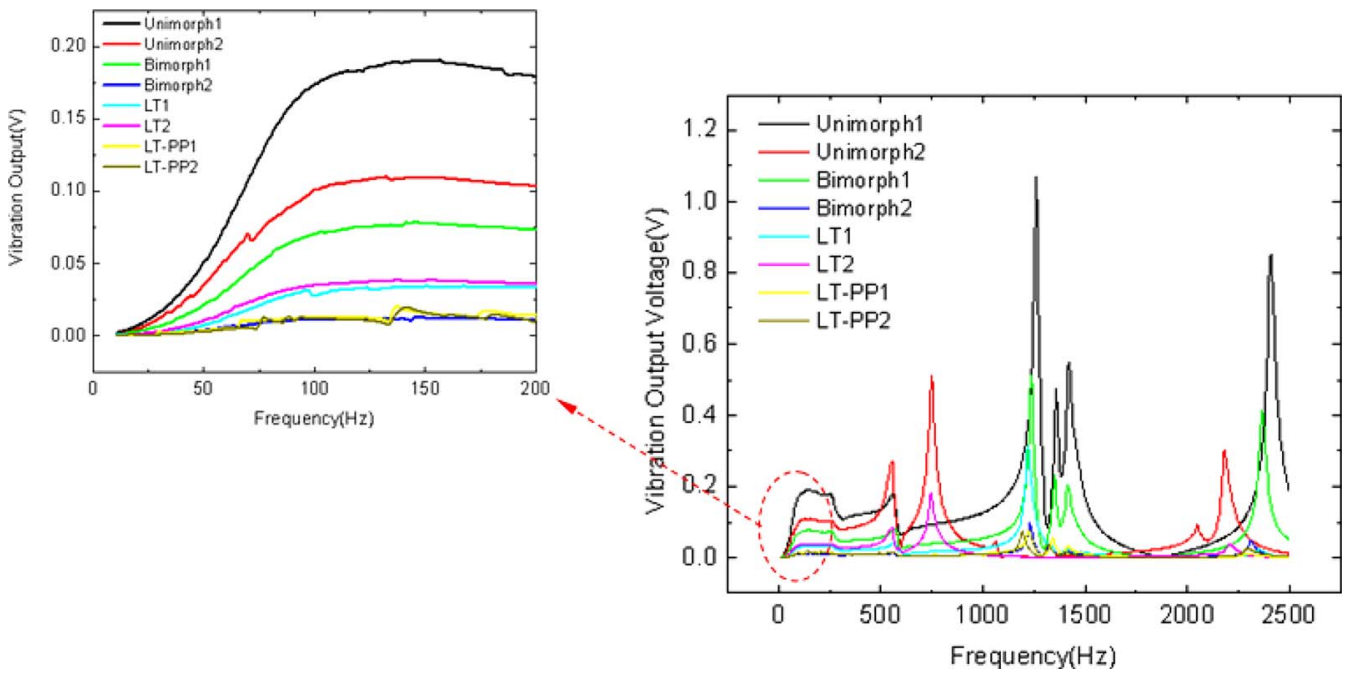

FIG. 13. (Color online) Vibrating damping experimental result for different ME sensor.

at the expense of decreased ranging capabilities. This, then, implies the second principle. To separate noise and the signal, a ME sensor should be designed such that the ME signal occurs in one mode, whereas the noise is constrained in another. Then, one can use a differential method to cancel the noise while not rejecting the signal. Third, certain laminate configurations have built-in vibration cancellation capabilities. These are the SS-U.S. (symmetrical signal, unsymmetrical noise) modes: such as L-T, L-L, T-T, T-L, and push-pull. If the environment that the sensor is required to operate in has a high degree of vibrations, one of these SS-UN modes is
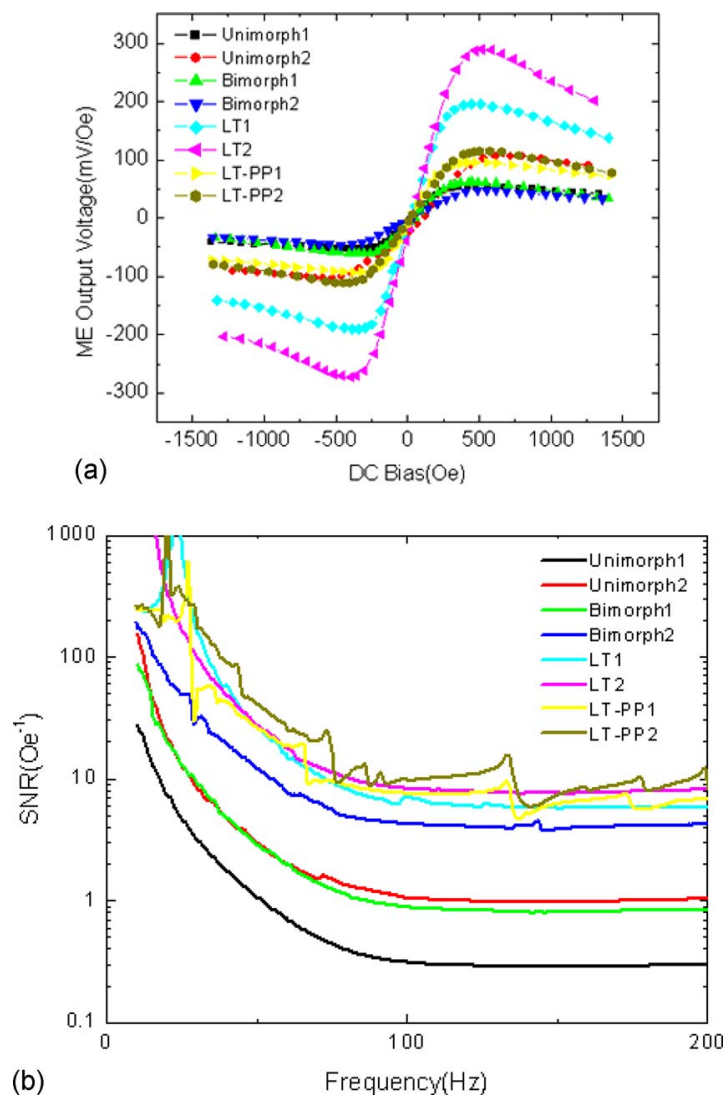

FIG. 14. (Color online) (a) ME voltage for different ME sensor and (b) SNR for different ME sensor. best suited for that environment. To further reduce vibrational noise, we recommend using laminates with a high stiffness in the transverse direction. This can be accomplished simply by use of a thicker ME laminate, if the sensor is limited to be thin, then we recommend using multilayer stacks or soft foam to absorb the vibrational energy. Fourth, in signal symmetrical structures, thermal noise cannot be rejected from the signal. This is simply because thermal noise is introduced, via longitudinal/transverse deformations, similar to the signal. Accordingly, U.S.-SN (unsymmetrical signal, symmetrical noise) modes have the ability to cancel thermal noise. If the environment in which the ME sensor operates is exposed to significant thermal changes on time scales of $>10^{-3} \mathrm{~s}$, then U.S.-SN mode ME laminate configurations are the best choice as magnetic sensors. Fifth, sometimes, it is hard to design the sensor with signal and noise mode separately, but we can still use a compromising design which set the signal mode stronger than that of the noise. Bimorph is such a good compromising example.

\section{ACKNOWLEDGMENTS}

The authors gratefully acknowledge the help of Professor Dan inman, Mr. Steve Anton, and Mr. Pablo Tarazaga from the center of Intelligent Material Systems and Structures, Virginia Tech.

\footnotetext{
${ }^{1}$ M. Fiebig, J. Phys. D 38, R123 (2005).

${ }^{2}$ M. I. Bichurin, V. M. Petrov, and G. Srinivasan, Phys. Rev. B 68, 054402 (2003).

${ }^{3}$ J. Ryu, A. V. Carazo, K. Uchino, and H. E. Kim, Jpn. J. Appl. Phys., Part 1 40, 4948 (2001).

${ }^{4}$ M. I. Bichurin, D. A. Filippov, V. M. Petrov, V. M. Laletsin, N. Paddubnaya, and G. Srinivasan, Phys. Rev. B 68, 132408 (2003).

${ }^{5}$ V. M. Petrov and G. Srinivasan, Phys. Rev. B 78, 184421 (2008).

${ }^{6}$ C. W. Nan, M. I. Bichurin, S. X. Dong, D. Viehland, and G. Srinivasan, J. Appl. Phys. 103, 031101 (2008).

${ }^{7}$ S. X. Dong, J. Y. Zhai, F. M. Bai, J. F. Li, D. Viehland, and T. A. Lograsso, J. Appl. Phys. 97, 103902 (2005).

${ }^{8}$ J. Y. Zhai, Z. P. Xing, S. X. Dong, J. F. Li, and D. Viehland, Appl. Phys. Lett. 93, 072906 (2008).

${ }^{9}$ Z. P. Xing, S. X. Dong, J. Y. Zhai, L. Yan, J. F. Li, and D. Viehland, Appl. Phys. Lett. 89, 112911 (2006).

${ }^{10}$ G. Srinivasan, C. P. De Vreugd, M. I. Bichurin, and V. M. Petrov, Appl.
} 
Phys. Lett. 86, 222506 (2005).

${ }^{11}$ J. G. Wan, Z. Y. Li, Y. Wang, M. Zeng, G. H. Wang, and J. M. Liu, Appl. Phys. Lett. 86, 202504 (2005)

${ }^{12}$ S. Dong, J. Zhai, F. Bai, J. F. Li, and D. Viehland, Appl. Phys. Lett. 87, 062502 (2005).

${ }^{13}$ W. M. Leach, Proc. IEEE 82, 1515 (1994).

${ }^{14}$ Z. P. Xing, J. F. Li, and D. Viehland, Appl. Phys. Lett. 91, 142905 (2007).
${ }^{15}$ Z. P. Xing, J. F. Li, and D. Viehland, Appl. Phys. Lett. 91, 182902 (2007). ${ }^{16}$ Z. P. Xing, J. Y. Zhai, J. Gao, J. F. Li, and D. Viehland, IEEE Electron Device Lett. 30, 445 (2009).

${ }^{17}$ S. X. Dong, J. Y. Zhai, Z. P. Xing, J. F. Li, and D. Viehland, Appl. Phys. Lett. 91, 022915 (2007).

${ }^{18}$ Z. P. Xing, J. Y. Zhai, S. X. Dong, J. F. Li, D. Viehland, and W. G. Odendaal, Meas. Sci. Technol. 19, 015206 (2008). 